\title{
Delayed febrile response with bloodstream infections in patients with continuous-flow left ventricular assist devices
}

\author{
Christopher A Wrobel, ${ }^{1}$ Mark H Drazner, ${ }^{2}$ Colby R Ayers, ${ }^{2}$ David D Pham, ${ }^{2}$ \\ Ricardo M La Hoz, ${ }^{3}$ Justin L Grodin, ${ }^{2}$ Sonia Garg, ${ }^{2}$ Pradeep P A Mammen, ${ }^{2}$ \\ Robert M Morlend, ${ }^{2}$ Faris Araj, ${ }^{2}$ Alpesh A Amin, ${ }^{2}$ William K Cornwell, ${ }^{2}$ \\ Jennifer T Thibodeau ${ }^{2}$
}

'Department of Internal Medicine, University of Texas Southwestern Medical Center, Dallas, Texas, USA ${ }^{2}$ Department of Internal Medicine, Division of Cardiology, University of Texas Southwestern Medical Center, Dallas, Texas, USA ${ }^{3}$ Department of Internal Medicine, Division of Infectious Disease, University of Texas Southwestern Medical Center, Dallas, Texas, USA

\section{Correspondence to} Dr Jennifer T Thibodeau, Department of Internal Medicine, Division of Cardiology, University of Texas Southwestern Medical Center, Dallas Texas 753909047, USA;

jennifer.thibodeau@ utsouthwestern.edu

Accepted 28 October 2018 Published Online First 29 January 2019

\section{ABSTRACT}

Bloodstream infections (BSIs) are common in patients with continuous-flow left ventricular assist devices (CF-LVADs). Whether CF-LVADs modulate the febrile response to BSIs is unknown. We retrospectively compared the febrile response to BSIs in patients with heart failure (HF) with CF-LVADs versus a control population of patients with $\mathrm{HF}$ receiving inotropic infusions. BSIs were adjudicated using the Centers for Disease Control and Prevention and the National Healthcare Safety Network criteria. Febrile status (temperature $\geq 38^{\circ} \mathrm{C}$, 100.4 ${ }^{\circ} \mathrm{F}$ ), temperature at presentation with BSI, and the highest temperature within 72 hours (Tmax) were collected. We observed 59 BSIs in LVAD patients and 45 BSIs in controls. LVAD patients were more likely to be afebrile and to have a lower temperature at presentation than control $(88 \%$ vs $58 \%, p=0.002$, and $37^{\circ} \mathrm{C} \pm 0.7$ vs $37.7^{\circ} \mathrm{C} \pm 1.0$, $\mathrm{p}=0.0009$, respectively). By 72 hours, the difference in afebrile status diminished ( $53 \%$ vs $44 \%, p=0.42$ ), and the Tmax was similar between the LVAD and control groups $\left(37.9^{\circ} \mathrm{C} \pm 0.9\right.$ vs $38.2^{\circ} \mathrm{C} \pm 0.8$, respectively, $\mathrm{p}=0.10)$. In conclusion, at presentation with a BSI, the vast majority of CF-LVAD patients were afebrile, an event which occurred at a higher frequency when compared with patients with advanced HF on chronic inotropes via an indwelling venous catheter. These data alert clinicians to have a very low threshold to obtain blood cultures in CFLVAD patients even in the absence of fever. Further study is needed to determine whether a delayed or diminished febrile response represents another pathophysiological consequence of CF-LVADs.

\section{INTRODUCTION}

Durable left ventricular assist devices (LVADs) are an accepted therapy for patients with advanced heart failure. ${ }^{1}$ However, they are associated with a number of complications, including right heart failure, stroke, device thrombosis, and gastrointestinal bleeding. ${ }^{12}$ Additionally, infectious complications occur commonly and lead to significant morbidity and mortality. ${ }^{1-11}$ In particular, bloodstream infections (BSIs),

\section{Significance of this study}

What is already known about this subject?

- Durable left ventricular assist devices (LVADs) are an accepted therapy for patients with advanced heart failure.

- LVADs are associated with a number of complications, including bloodstream infections (BSIs), which lead to significant morbidity and mortality.

- Prompt recognition of BSIs in patients with LVADs is important.

What are the new findings?

- The vast majority ( $88 \%$ ) of BSIs in LVAD patients were not associated with fever at time of presentation.

- The frequency of afebrile BSIs in the LVAD population was significantly greater than that of a control population of patients with advanced heart failure with indwelling lines for inotropic support.

- The average temperature at presentation with BSI was significantly lower in those with an LVAD compared with the control population.

How might these results change the focus of research or clinical practice?

- Validation studies are needed to confirm these novel findings.

- In continuous-flow LVAD (CF-LVAD) patients with subjective fever, chills, another source of infection, or an unexpected drop in blood pressure, clinicians should have a very low threshold to obtain blood cultures even if the patient is afebrile.

- Further study is needed to determine the mechanism of the delayed or diminished febrile response in patients with CFLVADs and whether it represents another pathophysiological consequence of continuous flow in the arterial circulation.

which occur in $12 \%-60 \%{ }^{1-578}$ of patients with LVADs, can progress to sepsis and have been associated with increased risk of ventricular 
assist device (VAD) thrombosis, ${ }^{911}$ stroke, ${ }^{610}$ and an in-hospital mortality in excess of 50\%. ${ }^{235}$ Thus, prompt recognition of BSIs is important.

Classically, fever is a presenting manifestation of BSIs. ${ }^{12}$ However, in our clinical experience, we noted LVAD patients with BSIs who were afebrile at the time of presentation. Thus, we undertook this study to evaluate the frequency of afebrile BSIs in patients with heart failure supported with an LVAD versus those on continuous infusions of an inotropic agent via an indwelling intravenous line. The latter was chosen as a control group as we reasoned such patients are known to be at risk of BSIs, as are patients supported with LVADs. Furthermore, the healthcare provider's threshold to draw blood cultures in patients either with an intravenous catheter, or an LVAD, likely would be lower as compared with patients without either indwelling device.

\section{METHODS}

This was a single-site, retrospective study of BSIs in patients with heart failure supported by a durable continuous-flow LVAD (CF-LVAD) versus those on chronic inotropic infusions. We retrospectively reviewed 188 consecutive patients who underwent CF-LVAD implantation at the University of Texas Southwestern Medical Center between 2009 and 2015. All patients included in this study were implanted with either a HeartMate II $(n=138)$ or HeartWare $(n=50)$ device. The control population of inotrope-dependent patients with advanced heart failure was identified using electronic medical record (EMR) discharge data, searching for patients discharged on inotropic support. A total of 631 patients had indwelling intravenous catheters for inotropic support between 2009 and 2015. Of these, 46 had BSIs and were included as the control group. As this was a retrospective study involving only chart review, informed consent was waived.

The EMRs of each patient were reviewed separately by two investigators. Demographic information was obtained. Events of interest included positive blood cultures from the time of LVAD implantation or indwelling line placement until December 31, 2016 or until death, transplant, line removal, or LVAD explantation. Patients with positive blood cultures were adjudicated as having a BSI using the Centers for Disease Control and Prevention and the National Healthcare Safety Network (CDC/NHSN) criteria: (1) the patient has a recognized pathogen cultured from one or more blood cultures, or (2) the patient has at least one of the following signs or symptoms: fever, chills, or hypotension, and common skin contaminant is cultured from two or more blood cultures drawn on separate occasions. ${ }^{13}$ For the second indication, the 'fever' criterion was met only if a documented fever was present at the time of bacteremia diagnosis. A subjective fever, where the patient stated that they felt as though they may have had a fever, did not meet this criterion.

Patients were defined as febrile at the time of BSI if the temperature measured closest to the time of blood culture draw was $\geq 38^{\circ} \mathrm{C}\left(100.4^{\circ} \mathrm{F}\right)$. The time the blood culture was obtained was determined by the 'collected' time documented in the culture result. It was also noted if patients were afebrile at the time blood cultures were drawn but developed a fever within the subsequent 72 hours. All patients who were febrile at the time of blood cultures or febrile in the subsequent 72 hours from the time of the blood culture were classified as 'febrile within 72 hours.' Subjective reports of fever did not impact classification of febrile status.

Clinical information for each BSI was also recorded, including temperature at time of blood cultures, maximum temperature within 72 hours of cultures (Tmax), white cell count at time of cultures, body mass index (BMI), if prescribed a daily aspirin or medication including acetaminophen, if on suppressive antibiotics, if had prior driveline infection, and reason for obtaining cultures.

\section{Statistical analysis}

Descriptive statistics for categorical variables are presented as count (percentage), and those for continuous variables are presented as mean \pm SD or median (25th, 75 th percentile) where appropriate. The Fisher's exact test was used to compare categorical data and the Kruskal-Wallis test to compare continuous variables for the per-person analysis. Generalized linear mixed modeling was used to assess the main effect of LVAD versus control and febrile versus afebrile. The generalized linear mixed modeling takes into account the correlated nature of the data, as these data are on a per-episode basis with multiple episodes per patient. A random intercept was used for each patient. Statistical significance was set at $p<0.05$. Statistical analyses were performed using SAS V.9.4.

\section{RESULTS}

There were 59 BSIs in 36 of 188 (19\%) LVAD patients, and 45 BSIs in 41 of $631(6 \%)$ controls. Eighteen additional episodes of positive blood cultures were not included (ten in LVAD patients and eight in control patients) as they did not meet the criteria for BSI as defined by the CDC/NHSN. Fourteen of the 36 LVAD patients had more than one BSI, and three of the 41 control patients had more than one BSI. Most patients in both groups were white, middle-aged men with a non-ischemic etiology of cardiomyopathy (table 1).

\section{Clinical characteristics during BSI episodes}

The clinical characteristics in the LVAD versus control patients at the time of BSIs are shown (table 2). In comparing the LVAD with the control patients, the former

\begin{tabular}{|c|c|c|c|}
\hline Characteristics & $\begin{array}{l}\text { LVAD } \\
\mathrm{n}=36\end{array}$ & $\begin{array}{l}\text { Control } \\
n=41\end{array}$ & $P$ values \\
\hline $\begin{array}{l}\text { Age at LVAD implantation or indwelling } \\
\text { line placement, years }\end{array}$ & $58 \pm 14$ & $55 \pm 13$ & 0.29 \\
\hline Male gender & $28(78)$ & $30(73)$ & 0.79 \\
\hline Race & & & 0.75 \\
\hline Caucasian & $19(53)$ & $23(56)$ & \\
\hline Black & $14(39)$ & $16(39)$ & \\
\hline Hispanic & $2(6)$ & $2(5)$ & \\
\hline Other & $1(3)$ & $0(0)$ & \\
\hline Ischemic etiology of cardiomyopathy & $11(31)$ & $13(32)$ & 1.0 \\
\hline
\end{tabular}

Data are presented as mean \pm SD or count (\%).

LVAD, left ventricular assist device. 
Table 2 Clinical characteristics of patients at time of bloodstream infection episodes

\begin{tabular}{llll}
\hline Characteristics & LVAD & Control \\
$\mathrm{n}=59$ & $\mathrm{n}=45$ & P values \\
\hline Age at BSI, years & $60 \pm 13$ & $55 \pm 13$ & 0.15 \\
\hline White cell count, $\times 10^{9}$ cells/L at time of culture & $9.3(6.8,14.4)$ & $9.7(7.2,13.0)$ & 0.49 \\
\hline MAP, $\mathrm{mm} \mathrm{Hg}$ at time of culture & $74(63,86)$ & $72(67,81)$ & 0.88 \\
\hline BMI, $\mathrm{kg} / \mathrm{m}^{2}$ & $27.5(23.0,32.5)$ & $27.7(25.1,32.0)$ & 0.52 \\
\hline Aspirin therapy & $47(80)$ & $23(51)$ & 0.02 \\
\hline Aspirin dose $325 \mathrm{mg}$ (vs $81 \mathrm{mg})$ & $29(63)$ & $6(25)$ & 0.02 \\
\hline Outpatient acetaminophen prescription & $14(24)$ & $7(16)$ & 0.44 \\
\hline Duration from LVAD placement or line to insertion to BSI, days & $303(151,628)$ & $32(12,92)$ & $<0.0001$ \\
\hline Duration from prior BSI episode to next episode, days (n=22 LVAD, $\mathrm{n}=4$ control) & $114(64,195)$ & $265(138,738)$ & 0.31 \\
\hline
\end{tabular}

Data are presented in mean \pm SD, median (25th, 75th percentile) or count (\%).

BMI, body mass index; BSI, bloodstream infection; LVAD, leftventricular assist device; MAP, mean arterial pressure.

were more likely to be afebrile $[52 / 59(88 \%)$ vs $26 / 45$ $(58 \%), p=0.002]$ and had a lower mean temperature at the time the blood cultures were obtained $\left(37.0^{\circ} \mathrm{C} \pm 0.7\right.$ vs $37.7^{\circ} \mathrm{C} \pm 1.0, \mathrm{p}=0.0009$ ), as shown in figure 1 . Twenty-one of the LVAD versus six of the control patients developed fever after the blood cultures were obtained, such that at 72 hours the difference in frequency of those who were afebrile between the two groups was no longer significant [31/59 (53\%) vs 20/45 (44\%), respectively, $\mathrm{p}=0.42]$, and the Tmax at 72 hours was similar between the LVAD and control groups $\left(37.9^{\circ} \mathrm{C} \pm 0.9\right.$ vs $38.2^{\circ} \mathrm{C} \pm 0.8$, respectively, $\mathrm{p}=0.10)$. When restricting the analysis to only those that developed fever, the mean temperature at time of culture remained lower in patients with LVADs versus control, and the Tmax within 72 hours was similar $\left(37.4^{\circ} \mathrm{C} \pm 0.8\right.$ vs $38.3^{\circ} \mathrm{C} \pm 0.7, p=0.0007$ and $38.7^{\circ} \mathrm{C} \pm 0.6$ vs $38.8^{\circ} \mathrm{C} \pm 0.6$, $\mathrm{p}=0.61$, respectively). For those who became febrile after the initial cultures were drawn, the median (25th, 75th percentile) time to fever was similar between the LVAD and control groups $(16$ hours $(7,31)$ vs 11 hours $(9,25)$, $\mathrm{p}=0.34$ ).

There was no difference in age, white blood cell count, mean arterial blood pressure, BMI, or use of an outpatient prescription containing acetaminophen at the time of the blood cultures in comparing the LVAD with control patients. However, those with LVADs were more likely to be prescribed a daily aspirin, and of those who were prescribed aspirin, LVAD patients were more likely to be prescribed $325 \mathrm{mg}$ daily rather than $81 \mathrm{mg}$ daily (table 2 ). Use of aspirin was evaluated with generalized linear mixed modeling to assess the main effect of LVAD versus control and febrile versus afebrile and was not significantly associated with febrile status $(p=0.36)$. Furthermore, when restricting the analysis to only those patients who were on aspirin, LVAD patients had lower mean temperature at the time of blood cultures compared with control patients $\left(36.9^{\circ} \mathrm{C} \pm 0.7\right.$ vs $37.7^{\circ} \mathrm{C} \pm 1.1$, respectively, $\left.\mathrm{p}=0.02\right)$ and were more likely to be afebrile [43/47 (91\%) vs $12 / 23$ $(52 \%), p=0.0004]$. C reactive protein, erythrocyte sedimentation rate, and procalcitonin levels were not available for most patients.

\section{Patients with more than one BSI}

Of the 14 LVAD patients with more than one BSI, nine patients were afebrile at the time of diagnosis with all BSIs (total of 19 episodes), and five LVAD patients were febrile at diagnosis in only one episode each, but afebrile in a total of 12 other episodes. There were only three patients in the control group who had more than one BSI. Of these patients, one patient had two febrile episodes, one had one febrile and one afebrile episode, and one had one febrile and two afebrile episodes.

When restricting our analysis to only the first BSI, LVAD patients were more likely to be afebrile at the time of BSI [32/36 (89\%) vs 25/41 (61\%), $\mathrm{p}=0.008]$ and to have
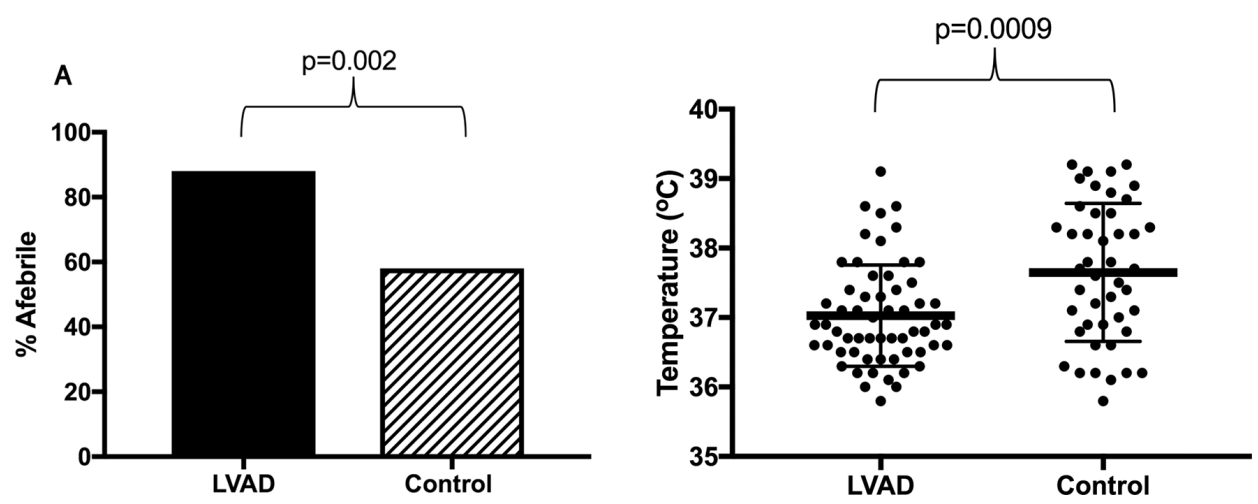

Figure 1 (A) Frequency of afebrile status at time of bloodstream infection. (B) Mean temperature at time of bloodstream infection. LVAD, left ventricular assist device. 
lower temperatures at the time of cultures $\left(37.0^{\circ} \mathrm{F} \pm 0.7\right.$ vs $37.6^{\circ} \mathrm{C} \pm 1.0, \mathrm{p}=0.009$ ).

\section{Reason for blood culture}

The most common reason for obtaining blood cultures was for documented (temperature recorded in the medical record) or subjective (patients stated that they felt as though they may have had a fever, although no temperature was taken) fever in 33 of 59 (56\%) LVAD BSIs and 24 of 45 (53\%) control BSIs. Among the 33 LVAD BSIs, 7 of 33 (21\%) had documented fever at the onset, 13 of 33 (39\%) had fever later in the BSI episode, and notably 13 of 33 (39\%) were never febrile. In the control population, 18 of $24(75 \%)$ had documented fever, 2 of $24(8 \%)$ became febrile later in the BSI episode, and 4 of 24 (17\%) were never febrile. Other common reasons for obtaining cultures in LVAD patients included concern for other infections such as a urinary tract infection or pneumonia $(5 / 59,8 \%)$, hypotension $(4 / 59,7 \%)$, and surveillance cultures done prior to transplant $(4 / 59,7 \%)$. Other common reasons in control patients included chills $(6 / 45,13 \%)$, surveillance cultures prior to LVAD or transplant $(5 / 45,11 \%)$, and leukocytosis $(3 / 45,7 \%)$. There was no protocol which led to LVAD patients having blood cultures drawn for a reason other than clinical suspicion for infection aside from our protocol to draw blood cultures when admitted prior to transplantation. Obtaining blood cultures immediately before transplant (or LVAD for the control population) is standard for all patients at our center, including patients with indwelling lines for inotropic support.

Clinical characteristics of LVAD patients by febrile status We next compared the clinical characteristics at the time of BSI in the LVAD patients, stratified by febrile status (table 3). There was no difference in age at time of BSI, gender, type of LVAD, duration from LVAD implantation to BSI, white blood cell count, mean arterial pressure at time of diagnosis, whether the patients were prescribed a daily aspirin or outpatient acetaminophen, or aspirin dosing in comparing the LVAD patients with fever versus without fever. Eighteen LVAD BSIs occurred while on suppressive antibiotics for either driveline infection $(n=14)$ or prior BSI $(n=4)$. However, use of suppressive antibiotics was evaluated with mixed modeling and was not associated with febrile status $(p=0.21)$. No control patients were receiving

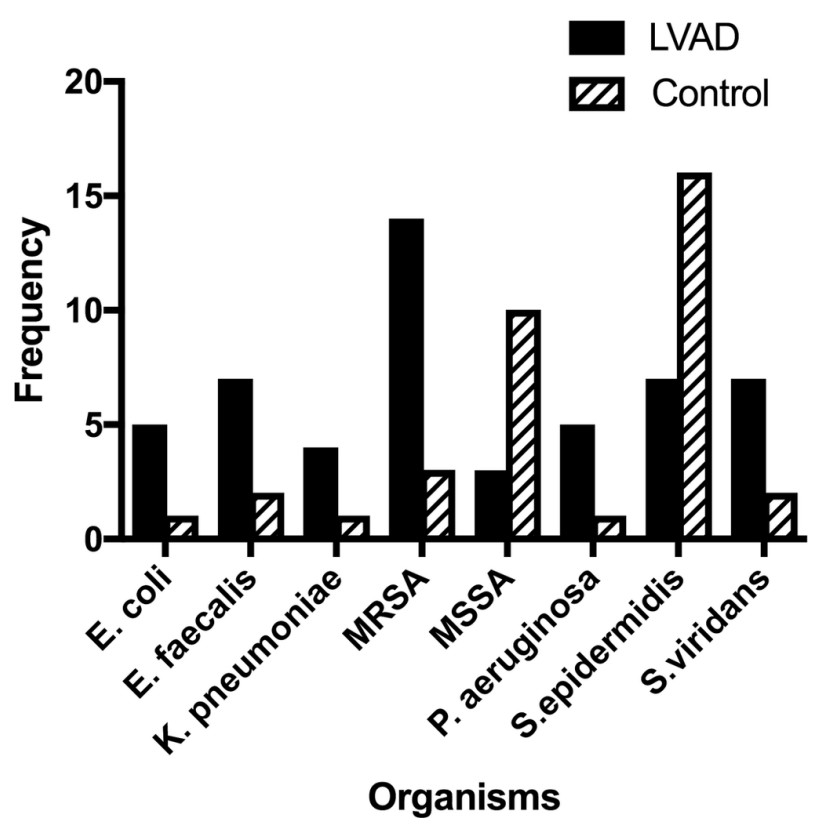

Figure 2 Bacterial pathogens of bloodstream infections. LVAD, left ventricular assist device; MRSA, methicillinresistant Staphylococcus aureus; MSSA, methicillinsensitive Staphylococcus Aureus.

antibiotics at the time of BSI. When restricting the analysis to only episodes where patients were not on suppressive antibiotic treatment, LVAD patients compared with control patients were still more likely to be afebrile [43/48 (90\%) vs 24/43 (56\%), respectively, $\mathrm{p}=0.003$ ] and had lower mean temperatures at the time blood cultures were drawn $(37.0 \pm 0.7$ vs $37.7 \pm 1.0, p=0.001)$.

\section{Bacterial pathogens}

The BSIs were due to a diverse spectrum of organisms (figure 2), particularly in the LVAD patients. There was a predominance of Gram-positive organisms isolated in both the LVAD and control groups (74\% vs 84\%). Methicillin-resistant Staphylococcus aureus (MRSA) was the most commonly isolated organism in the LVAD group, followed closely by $S$. epidermidis. S. epidermidis was the most common organism isolated in the control group, accounting for $33 \%$ of isolated organisms in this group. In

\begin{tabular}{llll}
\hline Table 3 & Clinical characteristics of LVAD patients at the time of bloodstream infection episodes, stratified by fever status \\
\hline & $\begin{array}{l}\text { Afebrile at time of culture } \\
\mathbf{n}=52\end{array}$ & $\begin{array}{l}\text { Febrile at time of culture } \\
\mathbf{n}=7\end{array}$ & \multicolumn{1}{c}{ P values } \\
\hline Characteristics & $60 \pm 13$ & $59 \pm 8$ & 0.42 \\
\hline Age at BSI, years & $299(147,624)$ & $587(285,628)$ & 0.31 \\
Duration from time from LVAD implantation to BSI, days & $32(62)$ & $6(86)$ & 0.43 \\
HeartMate II LVAD & $9.8(6.8,14.0)$ & $8.9(7.1,16.1)$ & 0.65 \\
White cell count, $\times 10^{9}$ cells/L at time of culture & $73(63,87)$ & $76(64,78)$ & 0.33 \\
MAP, mm Hg at time of culture & $27.4(22.8,31.8)$ & $32.5(23.1,37.7)$ & 0.12 \\
BMI, $\mathrm{kg} / \mathrm{m}^{2}$ & $43(83)$ & $4(57)$ & 0.20 \\
Aspirin therapy & $26(62)$ & $3(75)$ & 0.67 \\
\hline Aspirin dose $325 \mathrm{mg}$ (vs $81 \mathrm{mg})$ & $12(23)$ & $2(29)$ & 1.0 \\
Outpatient acetaminophen prescription & & \\
\hline
\end{tabular}

Data are presented in mean \pm SD, median (25th, 75th percentile) or count (\%).

$\mathrm{BMI}$, body mass index; BSI, bloodstream infection; LVAD, left ventricular assist device; MAP, mean arterial pressure. 
the LVAD patients, Escherichia coli,Pseudomonas aeruginosa, and Klebsiella pneumoniae were the most common Gram-negative organisms. There was no bacterial infection that was more likely to present with fever; however, those with MRSA infection were less likely to be febrile $(p=0.04)$.

\section{DISCUSSION}

This is the first study, to our knowledge, to suggest that patients supported with CF-LVADs have an altered clinical presentation in response to BSI. Specifically, we found a high frequency of afebrile BSIs (88\%) in the LVAD population, which was significantly greater than that of a control population (58\%). Additionally, the average temperature at presentation with BSI was significantly lower in those with an LVAD compared with the control population. Together, these findings suggest that healthcare providers need to consider bacteremia in patients with CF-LVADs even in the absence of fever.

Infectious complications in patients on mechanical support are common, particularly driveline infections and BSIs. ${ }^{1-578}$ When associated with sepsis, BSIs can convey an in-hospital mortality in excess of $50 \% .^{23}{ }^{27}$ Furthermore, infection increases the risk of VAD thrombosis and stroke $^{6-11}$; thus, prompt recognition of BSIs is essential in order to allow timely initiation of antibiotics and prevent potential complications from BSIs. We were unable to identify unique characteristics of those with LVAD who did or did not have fever at presentation with BSIs, suggesting that a heightened awareness of afebrile BSIs in all LVAD patients is warranted.

An important consideration is whether the BSIs in patients with LVADs were not true infections but rather artifactual from contamination of blood cultures. We do not believe this to be the case based on the pathogens identified (see figure 2), which included MRSA, E. coli, P. aeruginosa, and K. pneumoniae. Furthermore, BSIs due to $S$. epidermidis, which may be interpreted as a contaminant in some cases, were more common in the control population than in the LVADs. Additionally, bacteremic episodes were only included if they met the criteria for BSIs as delineated by the CDC/NHSN. ${ }^{13}$ In total, these data suggest that these episodes represented true BSIs.

The differences between the LVAD and control patients in frequency of fever had waned at 72 hours, as a numerically higher number of VAD patients $(40 \%$ vs $23 \%, p=0.21)$ developed fever after the blood cultures were obtained. These data suggest that some LVAD patients can ultimately mount a fever but at a delayed time point. However, given the importance of early treatment of BSIs, our finding of a delay in febrile status with BSI has clinical relevance.

We considered whether detection bias (ie, lower threshold to obtain blood cultures in those with an LVAD) could explain our findings. We do not believe this to be the case for several reasons. First, we deliberately chose a control group with indwelling central lines (for inotropes), reasoning that their predisposition to BSI would lower a healthcare provider's threshold to draw blood cultures, in a manner analogous to patients supported with an LVAD. Second, the majority of LVAD patients had other clinical manifestations to suggest BSI, including 56\% with documented or subjective fevers and $7 \%$ with hypotension.
Third, blood cultures were obtained for surveillance more commonly in the control group (11\%) than the LVAD group (7\%). Regardless, our data demonstrate that the vast majority of LVAD patients with BSIs are afebrile at the time of presentation and alert clinicians to have an extremely low threshold to obtain blood cultures for other symptoms (eg, subjective fever, hypotension, chills) even if there is no elevation in temperature. Specifically, if an LVAD patient reports subjective fever or chills, has another source of infection (eg, urinary tract infection or pneumonia), or has a drop in their blood pressure, evaluation for bacteremia should be initiated regardless of whether or not they have a documented fever.

Our investigation into the rate of afebrile BSIs developed from anecdotal patient experience, but we believe there is biologic plausibility to our findings. Patients with chronic heart failure have multiple immunologic derangements. ${ }^{14-17}$ In addition, LVAD recipients appear to develop further immune dysfunction after implantation via altered T-cell function, distortions in T-cell subtypes, and B-cell hyper-reactivity. ${ }^{18-21}$ We recognize that these findings were described in pulsatile LVADs, rather than contemporary CF-LVADs. However, to our knowledge there have not been studies investigating T-cell and B-cell function in CF-LVADs. Moreover, LVADs have been shown to be associated with a proinflammatory state, with elevated inflammatory markers and chemokines described in CF-LVAD patients. ${ }^{22-24}$ It is possible that chronically elevated cytokines could downregulate the thermoregulatory center, leading to less of a febrile response to an infection. Given the potential for an attenuated humoral and cellular response to bacterial antigens as well as the presence of a chronic inflammatory state, it is plausible that LVAD patients could have a diminished immunologic response and blunted thermoregulatory response to BSIs at presentation. Further mechanistic investigation is warranted.

There are several limitations to our study. This was a single-center study; however, the same two LVADs are used broadly throughout the world. Given the retrospective nature of this study, there may be unaccounted clinical factors that led differential practice patterns in obtaining blood cultures between the control and LVAD groups. However, we believed that patients with heart failure with indwelling intravenous catheters were the best comparator available given a lower than normal threshold to have blood cultures drawn, and because they also were patients with heart failure receiving hemodynamic support. The definition of a BSI by the CDC/NHSN included fever, chills, or hypotension as criteria if a patient presents with a common skin contaminant cultured from two or more blood cultures drawn on separate occasions. Because of this, we had to exclude 7 of 10 BSIs in VAD patients and 4 of 8 control patients due to afebrile status, as they did not otherwise meet the criteria for BSI. However, despite having to exclude a higher proportion of LVAD patients due to afebrile status, our findings persisted. Another potential limitation is that more LVAD patients were taking aspirin; however, we did not find aspirin use to be statistically significant in our mixed modeling assessment and the results were qualitatively unchanged when the analyses were restricted to those on aspirin therapy. Regardless of the underlying cause, these data demonstrate that patients 
with LVADs with BSIs in the clinical setting are usually afebrile at presentation.

In conclusion, patients with advanced heart failure supported by durable CF-LVADs versus those on chronic inotropic infusions have a lower temperature and higher frequency of being afebrile when they present with BSI, data which alert clinicians to have a very low threshold to obtain blood cultures in this patient population. Further investigation is needed to determine whether a reduced ability to mount a fever represents another pathophysiological consequence of CF-LVADs.

Contributors All authors have participated in this work and have reviewed and agree with the content of the article. The entire article has been written by the authors and they received no form of external sponsorship or honorarium. JT, CAW, MHD, RMLH, DDP, JLG, SG, PPAM, RMM, FA, AAA, and WKC were involved in the conception and design of the study and interpretation of data. CRA was involved in the interpretation of data. All authors were involved in the drafting or critical revision of the manuscript. All authors have given final approval of the submitted manuscript.

Funding MHD acknowledges support from the James M Wooten Chair in Cardiology. WKC receives funding from an NIH/NHLBI mentored patientoriented research career development award (1K23HL132048-01). RMLH receives support from the National Center for Advancing Translational Sciences of the National Institutes of Health under award number UL1TR001105. There are no relationships with industry.

Competing interests None declared.

Patient consent Not required.

Ethics approval The study protocol was reviewed and approved by the University of Texas Southwestern Institutional Review Board.

Provenance and peer review Not commissioned; externally peer reviewed.

Data sharing statement We do not have additional unpublished data to share.

\section{REFERENCES}

1 Kirklin JK, Naftel DC, Pagani FD, et al. Seventh INTERMACS annual report: 15,000 patients and counting. J Heart Lung Transplant 2015;34:1495-504.

2 Smedira NG, Hoercher KJ, Lima B, et al. Unplanned hospital readmissions after HeartMate II implantation: frequency, risk factors, and impact on resource use and survival. JACC Heart Fail 2013;1:31-9.

3 Toda K, Yonemoto Y, Fujita T, et al. Risk analysis of bloodstream infection during long-term left ventricular assist device support. Ann Thorac Surg 2012;94:1387-93.

4 Maniar S, Kondareddy S, Topkara VK. Left ventricular assist device-related infections: past, present and future. Expert Rev Med Devices 2011;8:627-34.

5 Nienaber JJC, Kusne S, Riaz T, et al. Clinical manifestations and management of left ventricular assist device-associated infections. Clin Infect Dis 2013;57:1438-48.

6 Trachtenberg BH, Cordero-Reyes AM, Aldeiri M, et al. Persistent blood stream infection in patients supported with a continuous-flow left ventricular assist device is associated with an increased risk of cerebrovascular accidents. J Card Fail 2015;21:119-25.

7 John R, Aaronson KD, Pae WE, et al. Drive-line infections and sepsis in patients receiving the HVAD system as a left ventricular assist device. J Heart Lung Transplant 2014;33:1066-73.

8 Topkara VK, Kondareddy S, Malik F, et al. Infectious complications in patients with left ventricular assist device: etiology and outcomes in the continuousflow era. Ann Thorac Surg 2010;90:1270-7.

9 Uriel N, Han J, Morrison KA, et al. Device thrombosis in HeartMate II continuous-flow left ventricular assist devices: a multifactorial phenomenon. J Heart Lung Transplant 2014;33:51-9.

10 Frontera JA, Starling R, Cho SM, et al. Risk factors, mortality, and timing of ischemic and hemorrhagic stroke with left ventricular assist devices. I Heart Lung Transplant 2017;36:673-83.

11 Kirklin JK, Naftel DC, Kormos RL, et al. Interagency Registry for Mechanically Assisted Circulatory Support (INTERMACS) analysis of pump thrombosis in the HeartMate II left ventricular assist device. J Heart Lung Transplant 2014;33:12-22.

12 McCulloh RJ. Opal SM. Bacteremia and Sepsis. In: Benjamin IJ, Griggs RC, Wang EJ, Fitz JG, eds. Andreoli and Carpenter's Cecil Essentials of Medicine. 9th edn. Philadelphia, PA: Elsevier Saunders, 2016:846-52.

13 Horan TC, Andrus M, Dudeck MA. CDC/NHSN surveillance definition of health care-associated infection and criteria for specific types of infections in the acute care setting. Am J Infect Control 2008;36:309-32.

14 Levine B, Kalman J, Mayer L, et al. Elevated circulating levels of tumor necrosis factor in severe chronic heart failure. N Eng/ J Med 1990;323:236-41.

15 Munger MA, Johnson B, Amber IJ, et al. Circulating concentrations of proinflammatory cytokines in mild or moderate heart failure secondary to ischemic or idiopathic dilated cardiomyopathy. Am J Cardiol 1996;77:723-7.

16 Testa M, Yeh M, Lee P, et al. Circulating levels of cytokines and their endogenous modulators in patients with mild to severe congestive heart failure due to coronary artery disease or hypertension. J Am Coll Cardiol 1996;28:964-71.

17 Anker SD, von Haehling S. Inflammatory mediators in chronic heart failure: an overview. Heart 2004;90:464-70.

18 Itescu S, Ankersmit JH, Kocher AA, et al. Immunobiology of left ventricular assist devices. Prog Cardiovasc Dis 2000;43:67-80.

19 Itescu S, John R. Interactions between the recipient immune system and the left ventricular assist device surface: immunological and clinical implications. Ann Thorac Surg 2003;75:S58-S65.

20 Ankersmit HJ, Tugulea S, Spanier T, et al. Activation-induced T-cell death and immune dysfunction after implantation of left-ventricular assist device. Lancet 1999;354:550-5.

21 Ankersmit HJ, Edwards NM, Schuster M, et al. Quantitative changes in T-cell populations after left ventricular assist device implantation: relationship to T-cell apoptosis and soluble CD95. Circulation 1999;100:II-211-0.

22 Grosman-Rimon L, McDonald MA, Jacobs I, et al. Markers of inflammation in recipients of continuous-flow left ventricular assist devices. Asaio J 2014;60:657-63.

23 Caruso R, Verde A, Campolo J, et al. Severity of oxidative stress and inflammatory activation in end-stage heart failure patients are unaltered after 1 month of left ventricular mechanical assistance. Cytokine 2012;59:138-44.

24 Grosman-Rimon L, Jacobs I, Tumiati LC, et al. Longitudinal assessment of inflammation in recipients of continuous-flow left ventricular assist devices. Can J Cardiol 2015;31:348-56. 